\title{
GEE-smoothing spline in semiparametric model with correlated nominal data
}

\begin{abstract}
In this paper we propose GEE-Smoothing spline in the estimation of semiparametric models with correlated nominal data. The method can be seen as an extension of parametric generalized estimating equation to semiparametric models. The nonparametric component is estimated using smoothing spline specifically the natural cubic spline. We use profile algorithm in the estimation of both parametric and nonparametric components. The properties of the estimators are evaluated using simulation studies.
\end{abstract}

Keyword: Generalized estimating equation; Nominal data; Properties of estimator; Smoothing spline; Simulation study 